

\section{ÍNDICE}

\section{ÁMBITOS PERSONALES PERSONALÁMBITOS}

Crisis de Venezuela: Análisis y perspectivas según los titulares de la prensa argentina, española y china

Venezuela's crisis: Analysis and perspectives in the headlines of Argentine, Spain and China press

Hui Feng Liu

Tratamiento informativo de la violencia de género: asesinatos de mujeres. Análisis de la agencia EFE

Informative treatment of gender violence: murders of women. Analysis of the press agency EFE Rosa Rodríguez Cárcela, Agustín López Vivas

MONOGRAFICOS MONOGRAPHS

Presentación Monográfico. El universo transmedia de los medios de comunicación universitarios: acción dentro y fuera del aula en la sociedad postdigital

Antonia Isabel Nogales-Bocio, Ángels Álvarez villa

El papel transformador de la radio universitaria en materias teóricas ajenas a la comunicación

The transformative role of college radio in theoretical subjects outside communication

Miguel Ángel Díaz Monsalvo

La radiodifusión universitaria: acción discursiva radiofónica para la divulgación de la ciencia University Radio Broadcasting: Radiophonic Discursive Action for the Science Popularization Jorge Sadi Durón, Joel Zapata Salazar

El uso corporativo de Instagram en las universidades privadas españolas. Estudio comparativo de treinta y cinco universidades

The corporate use of Instagram in spanish private universities. Comparative analysis of thirty-five private universities 
La radio universitaria como herramienta de inclusión social: OndaCampus en contextos como la cárcel y barrios desfavorecidos

The university radio as a tool for social inclusion: OndaCampus in contexts like the prison and disadvantaged neighborhoods

Leonor Real Adame, Daniel Martín-Pena, Macarena Parejo Cuéllar

Hacer radio universitaria en la era de YouTube: uso de la plataforma de vídeos a demanda en el contexto mexicano

Make college radio in the age YouTube: use of video on demand platform in the Mexican context Marina Vázquez Guerrero

\section{ARTÍCULOS ARTICLES}

O impresso e o digital nos modelos de negócios de jornais locais: uma análise do Sermos Galiza

The press and digital in business models from local media: Analysis of newspaper Sermos Galiza

Giovanni Ramos

Publicidad y cáncer en la prensa escrita (1903-1912)

Advertising and cancer in the written press (1903-1912)

Laura Almudéver-Campo, Ramón Camaño-Puig

Game rules vs. fandom. How Nintendo's Animal Crossing fan-made content negotiates the videogame meanings

Las reglas del juego vs. el fandom. Cómo el contenido hecho por fans de Nintendo Animal Crossing negocia los significados del videojuego

Jose A. Moreno

La comedia de situación y su análisis textual: evolución de los elementos constructivos del formato

The sitcom and its textual analysis: evolution of the constructive elements of the format

Darío Martín Sánchez

Análisis del discurso emocional de Donald Trump en la campaña electoral de $\mathbf{2 0 1 6}$ Analysis of Donald Trump's emotional speech on the 2016 election campaign 
Innovar, comunicar y transformar (en) la Universidad

Innovate, communicate and transform (at) the University

María Sofía Bernat

288-292

Transparencia en los medios: Un requisito imprescindible para medir la rentabilidad social en radio y televisión

Transparency in the media: An essential requirement to measuresocial profitability in radio and television

Amanda Salazar

Los estudios feministas en comunicación: representación de las mujeres en la revolución tecnológica

Feminist studies in communication: representation of women in the technological revolution 


\title{
Innovar, comunicar y transformar (en) la Universidad
}

\author{
Innovate, communicate and transform (at) the University
}

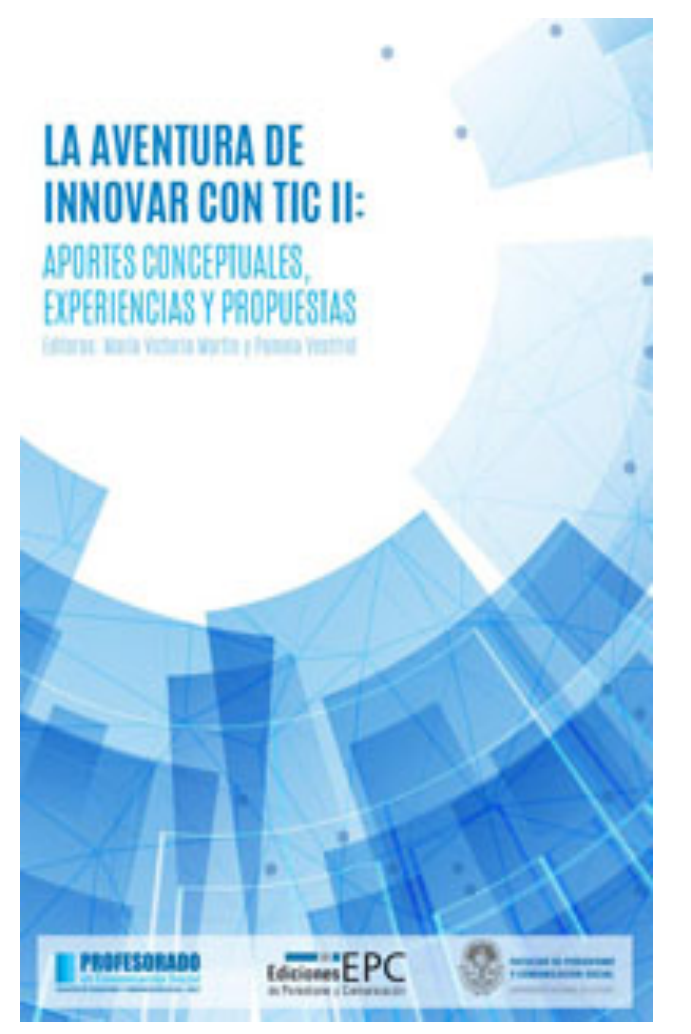

La aventura de innovar con TIC II: aportes

conceptuales, experiencias y propuestas

María Victoria Martin y Pamela Vestfrid

Ediciones de Periodismo y Comunicación

(EPC), La Plata, 2018

175 páginas

Reseña por María Sofía Bernat

DOI: http://dx.doi.org/10.12795/Ambitos.2020.i47.14

Omar Rincón (en Martin y Vestfrid, 2018, p. 10) sostiene que en los últimos tiempos emerge otra lógica de comunicación y ocurre una "re-invención que ha dejado atrás la

\section{Forma de citar:}

Bernat, M. S. (2020). Innovar, comunicar y transformar (en) la Universidad. Ámbitos. Revista Internacional de Comunicación 47, pp. 288-292. doi: 10.12795/Ambitos.2020.i47.14 
hegemonía escritural-libresca para pasar [a] un estallido de escrituras que se guían más por una lógica oral-visual. Lo que han aparecido son nuevas formas de escribir, nuevos lenguajes, otras maneras de comunicar el mundo". Y lo dice en el prólogo de "La aventura de innovar con TIC II: aportes conceptuales, experiencias y propuestas", un libro elaborado en el marco del Taller de Estrategias de Trabajo Colaborativo en Redes Sociales Virtuales y Otros Asistentes Online, dictado por las profesoras María Victoria Martin y Pamela Vestfrid desde hace cinco años en la Facultad de Periodismo y Comunicación Social de la Universidad Nacional de La Plata (UNLP). Esa aseveración en tal contexto es fundamental: pues traza una propuesta de lectura de un material que se vuelve indispensable si creemos que la comunicación y la educación son procesos inmersos en contextos que los resignifican, complejizan y retroalimentan y que, en consecuencia, se pueden transformar.

De acuerdo a sus editoras, se trata del segundo volumen digital realizado por los/as docentes de la cátedra y los/as estudiantes, que tiene como objetivo "problematizar en sentido amplio la vinculación entre la educación y las pantallas (...). Se busca promover la reflexión teórica y práctica relativa a la incorporación de tecnologías digitales para los diferentes perfiles del estudiantado" (Martin y Vestfrid, 2018, p. 4) de la unidad académica: periodistas, planificadores/as, profesores/as.

El libro, que está disponible en versión digital en este enlace, consta de cuatro ejes temáticos: el marco político-legal relacionado a normativas comunicacionales y educativas; el marco empírico concreto; recursos abiertos o asistentes; y modelos colaborativos y su vinculación con las prácticas profesionales de comunicadores y comunicadoras. Un punto a destacar es que se puede seguir -o inventar- cualquier orden de lectura para poner en discusión diferentes aspectos de las tecnologías. Es decir, la propuesta es creativa no sólo en sus contenidos, sino en los modos de lectura $-\mathrm{y}$, por ende, de producción de sentidos- que habilita.

Más allá de los ejes mencionados, esta publicación se divide en seis partes atravesadas por tales cuestiones.

En la primera, se reflexiona sobre las mediaciones de las Tecnologías de la Información y la Comunicación (TIC), la educación, el rol de educadores/as, el empleo de buscadores académicos y repositorios para comprender las formas de acceder a los saberes en la actualidad y los procesos pedagógicos en contextos de convergencia. Sobre este asunto, se afirma que es un fenómeno cultural que "modificó los modos de circulación y acceso al conocimiento, de relación entre los sujetos y por lo tanto de constitución de la subjetividad" (Novomisky y Manccini en Martin y Vestfrid, 2018, p. 28).

En un segundo apartado, denominado "Sujetos y contextos digitales", se presentan producciones desarrolladas por los y las estudiantes durante la cursada del año 2016, 
es decir, que en todo lo que sigue se incluyen dichas realizaciones. A partir de distintos recursos, como nubes de etiquetas, historietas, mapas conceptuales y posters, se ponen de relieve temáticas abordadas en la facultad: el lenguaje multimedial, la creatividad, la reconfiguración de comunicadores/as, el uso de TIC en educación, juventudes y medios de comunicación, planificación estratégica, criminología mediática, redes sociales virtuales, etc.

En la tercera parte, se analiza el trabajo colaborativo: su división de roles equitativa, la diversidad presente en el grupo, los objetivos compartidos, la importancia de los recursos informáticos para realizar esta tarea (Facebook, Google Drive, Prezi, etc), las diferencias entre trabajo colaborativo y grupal, la articulación de saberes, las responsabilidades, la relevancia de la comunicación, la enseñanza/aprendizaje de este enfoque, entre otros aspectos. También se vislumbran las maneras en las que las TIC reconfiguran las prácticas comunicacionales, se presentan modelos teóricos para comprender procesos posibilitados por las tecnologías en la vida cotidiana, como el modelo TPACK (en español, Conocimiento Tecnológico Pedagógico Disciplinar) y la taxonomía de Bloom, que propone el aprendizaje en tanto proceso escalonado. A su vez -y como comentamos en líneas anteriores- se incluyen producciones resultado del trabajo colaborativo efectuadas por los/as estudiantes. En otro apartado, siguiendo a las cursantes del taller Ivana Alzugaray, Noelia Deguer y Constanza Mackrey (en Martin y Vestfrid, 2018, p. 142), se señala que este tipo de trabajo "nos enseña a mejorar conductas y a enriquecernos en la interacción con el otro porque negociamos la palabra, consensuamos y asumimos la responsabilidad en conjunto. Aprendemos colaborativamente y valoramos el compromiso y el trabajo de cada uno de los integrantes".

La cuarta sección está dedicada al análisis de la legislación existente en Argentina en materia de comunicación y educación. Se menciona la Ley de Servicios de Comunicación Audiovisual (2009), el posterior Decreto de Necesidad y Urgencia a partir del cual fue derogada (2015) y la Ley de Educación Nacional (2006). Por otra parte, se plantean las potencialidades y los problemas derivados de los cambios de leyes. Al respecto, tres estudiantes dan cuenta de un caso concreto, la creación del canal televisivo de la Universidad Nacional de La Plata, al que definen como "un hijo de la Ley de Servicios de Comunicación Audiovisual", que posibilita a las distintas unidades académicas la generación de contenidos y discursos en manos de sus protagonistas. Además, señalan que cuantiosos/as estudiantes de la Facultad de Periodismo y Comunicación Social (UNLP) "utilizan ese espacio como práctica preprofesional y hoy en día por el desfinanciamiento que esta señal televisiva está sufriendo por parte del Estado, se encuentra en duda su continuidad" (Darguibel, Gómez y Ziaurruiz en Martin y Vestfrid, 2018, p. 119).

En quinto lugar se aborda la ya mencionada taxonomía de Bloom y el modelo TPACK para problematizar sobre los modos de participación, el empoderamiento social, los 
medios de comunicación tradicionales y los comunitarios y las posibilidades desplegadas a partir del uso de TIC. Por ejemplo, las estudiantes Florencia Gamón, Julia Pazzi y María Paula Rodríguez (en Martin y Vestfrid, 2018, p. 152) afirman: "Los medios comunitarios, al no tener el mismo espacio que los medios privados, utilizan las redes sociales como medio para difundir su mirada de la realidad en representación de los sectores que reivindican".

Por último, en la sexta parte, se imaginan "recorridos posibles". Los/as estudiantes problematizan la idea de comunicar en la era digital y, además, analizan la cursada de este taller a partir de los conceptos de TIC, TAC (Tecnologías del Aprendizaje y el Conocimiento), TEP (Tecnologías del Empoderamiento y Participación), del trabajo colaborativo y del modelo TPACK, destacando el carácter innovador de este modo de enseñanza/aprendizaje acaecido en la facultad.

Si bien las secciones parecen fijas, se superponen y relacionan estos temas y problemáticas a lo largo de todo el libro, es decir, no se presentan dichas cuestiones de manera aislada, sino que se buscan sus articulaciones y yuxtaposiciones.

Es interesante subrayar que en esta publicación la perspectiva de derechos atraviesa todas las discusiones y producciones.

Asimismo, se analiza cómo las TIC producen transformaciones en los modos de actuar y de pensar, pero no de manera determinista, sino que se reconoce la agencia de los sujetos y las relaciones entre lo online y lo offline.

La mirada también se ubica en las políticas públicas educativas (por ejemplo, programas como "Conectar Igualdad" en Argentina que posibilitaba el acceso a netbooks a miles de estudiantes en todo el país) y científicas (como la incorporación en repositorios digitales con acceso abierto de las investigaciones latinoamericanas emprendidas con fondos públicos), en una búsqueda constante de democratizar la educación y la ciencia y, por lo tanto, de contribuir a que nuestras sociedades sean más justas y equitativas.

Para finalizar, queremos destacar la importancia -y el desafío- de (co)producir un libro con estudiantes, de hacer públicas las miradas y puntos de vista de los actores intervinientes en lo que se erige como un proceso de enseñanza/aprendizaje aún en esta instancia. Aquí aparece claramente la pretensión de construir pedagogías solidarias, donde se ponen en cuestión asimetrías y desigualdades -que siempre están presentes- y se comparten modos de producir, de decir y de reflexionar, sin que ello implique un punto de vista homogéneo sobre las cuestiones abordadas. En el prólogo, Rincón afirma que es preciso intentar que la Universidad se convierta en un espacio de experimentación y de error, que hay que evitar que los y las estudiantes se parezcan a "nosotros" (los/as docentes con saberes profesionales y, por lo tanto, 
legitimados) o construyan conocimientos útiles únicamente para el mercado. Agrega que es necesario crear otros modelos de " prototipos ideales de para qué formamos en la Universidad: uno que se pone en riesgo, busca otras relaciones, cuenta en cuanto experimenta, se equivoca y encuentra su forma en el mundo. Más fracasar, menos saberes académicos, otros modos al mercado" (Rincón en Martin y Vestfrid, 2018, p. 19). De esta manera, a pesar de que remarcamos las discusiones que abre esta publicación, sí hay un aspecto que se asume irrenunciablemente: la comunicación y la educación son derechos, no mercancías. Y este libro pone de relieve el potencial transformador de dicho campo: del statu quo, del mundo, de nuestras prácticas cotidianas y sentidos y, por supuesto, de los sujetos. 